1. Suárez C, Rodrigo JP, Rinaldo A, Langendijk JA, Shaha AR, Ferlito A. Current treatment options for recurrent nasopharyngeal cancer. Eur Arch Otorhinolaryngol. 2010;267(12):1811-1824.

2. Liu S-C, et al. Leukemia inhibitory factor promotes nasopharyngeal carcinoma progression and radioresistance. J Clin Invest. 2013;123(12):5269-5283.

3. Mathieu ME, et al. LIF-dependent signaling: new pieces in the Lego. Stem Cell Rev. 2012;8(1):1-15.

4. Kamohara H, Ogawa M, Ishiko T, Sakamoto K, Baba H. Leukemia inhibitory factor functions as a growth factor in pancreas carcinoma cells: Involvement of regulation of LIF and its receptor expression. Int J Oncol. 2007;30(4):977-983.

5. Estrov $Z$, et al. Leukemia inhibitory factor binds to human breast cancer cells and stimulates their proliferation. J Interferon Cytokine Res. 1995;15(10):905-913

6 . Peñuelas $S$, et al. TGF- $\beta$ increases glioma-initiating cell self-renewal through the induction of LIF in human glioblastoma. Cancer Cell. 2009; 15(4):315-327.

7. Arthan D, Hong SK, Park JI. Leukemia inhibitory factor can mediate Ras/Raf/MEK/ERK-induced growth inhibitory signaling in medullary thyroid cancer cells. Cancer Lett. 2010;297(1):31-41.

8. Nonoyama M, Pagano JS. Homology between Epstein-Barr virus DNA and viral DNA from Burkitt's lymphoma and nasopharyngeal carcinoma determined by DNA-DNA reassociation kinetics. Nature. 1973;242(5392):44-47.

9. Raab-Traub N, Flynn K. The structure of the termini of the Epstein-Barr virus as a marker of clonal cellular proliferation. Cell. 1986;47(6):883-889.

10. Miller WE, Cheshire JL, Baldwin AS, Baldwin AS Jr, Raab-Traub N. The NPC derived C15 LMP1 protein confers enhanced activation of NF- $\mathrm{KB}$ and induction of the EGFR in epithelial cells. Oncogene. 1998;16(14):1869-1877.

11. Scholle F, Bendt KM, Raab-Traub N. Epstein-Barr virus LMP2A transforms epithelial cells, inhibits cell differentiation, and activates Akt. J Virol. 2000;74(22):10681-10689.

12. Shair KH, Raab-Traub N. Transcriptome changes induced by Epstein-Barr virus LMP1 and LMP2A in transgenic lymphocytes and lymphoma. MBio. 2012;3(5):e00288-12.

13. Dawson CW, Port RJ, Young LS. The role of the EBV-encoded latent membrane proteins LMP1 and LMP2 in the pathogenesis of nasopharyngeal carci- noma (NPC). Semin Cancer Biol. 2012;22(2):144-153. 14. Liu MT, et al. Epstein-Barr virus latent membrane protein 1 induces micronucleus formation, represses DNA repair and enhances sensitivity to DNA-damaging agents in human epithelial cells. Oncogene. 2004;23(14):2531-2539.

15. Gruhne B, Sompallae R, Masucci MG. Three EpsteinBarr virus latency proteins independently promote genomic instability by inducing DNA damage, inhibiting DNA repair and inactivating cell cycle checkpoints. Oncogene. 2009;28(45):3997-4008.

16. Tamm C, Böwer N, Annerén C. Regulation of mouse embryonic stem cell self-renewal by a YesYAP-TEAD2 signaling pathway downstream of LIF. J Cell Sci. 2011;124(pt 7):1136-1144.

17. Zeng Q, Hong W. The emerging role of the hippo pathway in cell contact inhibition, organ size control, and cancer development in mammals. Cancer Cell. 2008;13(3):188-192.

18. Bao S, et al. Glioma stem cells promote radioresistance by preferential activation of the DNA damage response. Nature. 2006;444(7120):756-760.

19. Chien YC, et al. Serologic markers of Epstein-Barr virus infection and nasopharyngeal carcinoma in Taiwanese men. NEnglJ Med. 2001;345(26):1877-1882.

\title{
Tuning mTOR activity for immune balance
}

\author{
Kai Yang and Hongbo Chi
}

Department of Immunology, St. Jude Children's Research Hospital, Memphis, Tennessee, USA.

\begin{abstract}
The mTOR pathway orchestrates diverse physiological processes, including $T$ cell functions and fate decisions; however, the regulation of mTOR-dependent $T$ cell differentiation remains elusive. In this issue, Park et al. examine the role of TSC1, an mTOR signaling regulator, in T cell differentiation and the balance between $T$ cell-mediated immunity and tolerance. They found that enhanced mTOR activity in Tsc1-deficient T cells promotes Th1 and Th17 differentiation, leading to increased intestinal inflammation in murine colitis. Tsc1-deficient Tregs had impaired suppressive activity in inflammatory conditions. These defects were associated with the acquisition of effector-like phenotypes and could be further exacerbated by concomitant loss of transcription factor Foxo3. This study highlights that TSC1-mediated control of mTOR activity impinges on the balance between immunity and tolerance by dictating effector and regulatory $T$ cell responses.
\end{abstract}

\section{Introduction}

mTOR is an evolutionarily conserved serine/threonine kinase that couples diverse cellular and environmental cues to cell growth, proliferation, and differentiation. In mammalian cells, mTOR interacts with multiple proteins and forms two distinct complexes, mTOR complex 1 (mTORC1) and mTORC2, which exhibit distinct roles in many physiological processes (1). The scaffold proteins Raptor and Rictor are characterized as obligatory

Conflict of interest: The authors have declared that no conflict of interest exists.

Citation for this article: J Clin Invest. 2013; 123(12):5001-5004. doi:10.1172/JCI73202. components of mTORC1 and mTORC2, respectively (Figure 1). mTORC1 activity is tightly controlled by multiple regulators. The upstream TSC complex, composed of tuberous sclerosis complex 1 (TSC1) and TSC2, inhibits mTORC1 activity by suppressing the function of Rheb (Ras homolog enriched in brain). Mutations of TSC1 or TSC 2 are associated with hamartoma syndrome with tissue overgrowth. Furthermore, mTOR dysfunction contributes to a large number of human diseases, including cancer, obesity, type 2 diabetes, and neurodegeneration (1).

In the immune system, $T$ cells play a pivotal role in adaptive immunity. Emerging evidence reveals that mTOR signaling impinges on multiple physiological processes of $\mathrm{T}$ cells, including their development, homeostasis, proliferation, and differentiation (2). Disruption of mTORC2 by ablation of Rictor impairs Notch-mediated proliferation and differentiation of pre-T cells in thymus $(3,4)$ and delays malignant transformation in a murine model of $\mathrm{T}$ cell acute lymphoblastic leukemia (T-ALL) (3). In the peripheral immune compartment, mTOR-deficient $\mathrm{CD}^{+}{ }^{+} \mathrm{T}$ cells are unable to differentiate into effector cells, including Th1, Th2, and Th17 cells, and instead preferentially develop into induced Tregs (5). Furthermore, Rheb/mTORC1 and mTORC2 are selectively involved in the lineage differentiation of effector $T$ cells $(6,7)$. Aside from these recent advances in our understanding of the core machinery of mTOR signaling, we are also starting to appreciate the $T$ cell-modifying effects of dysregulated mTOR activity via the genetic modulation of mTOR upstream regulators (2). Under steady state, TSC1 deficiency and the ensuing mTORC1 activation disrupt the quiescence of naive $T$ cells, leading to defective cell survival and antibacterial immune responses (8-10). Thus, keeping mTORC1 in check is essential for the maintenance of $\mathrm{T}$ cell homeostasis; however, the function of 
A
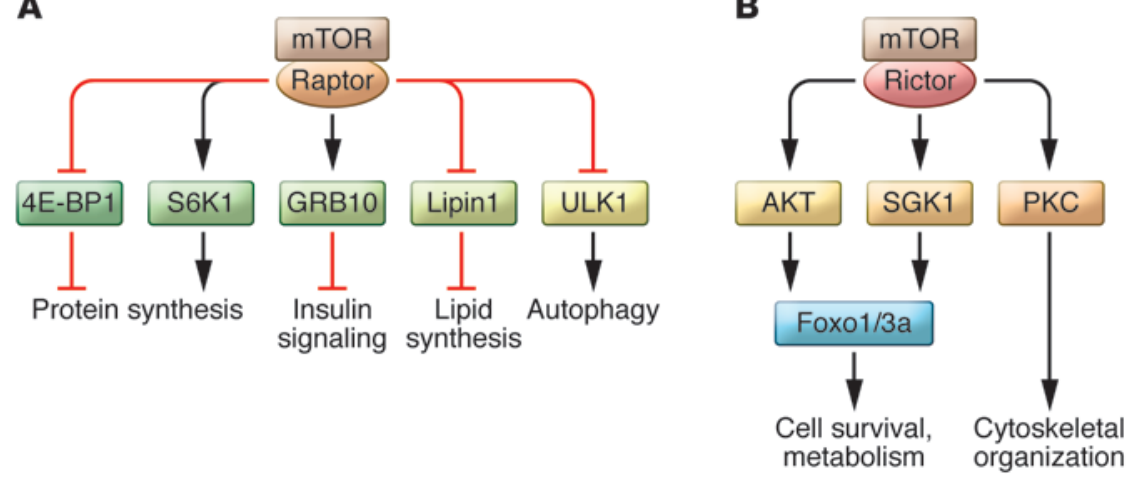

Figure 1

Schematics of mTOR signaling. mTORC1 and mTORC2 signal through distinct downstream effector pathways to orchestrate diverse cellular processes.

TSC1 in $\mathrm{CD}^{+} \mathrm{T}$ cell fate decisions and inflammatory responses remains largely unexplored.

\section{Control of Th1 and Th17 differentiation and intestinal inflammation}

Effector $\mathrm{T}$ cells are crucial for protective immunity against pathogens in the mucosal environment, but their excessive activity may drive inflammation. Park et al. observed that aged mice (over 20 weeks old) with T cell-specific ablation of TSC1 developed spontaneous inflammation and lymphocyte infiltration in the colon and liver (11), suggesting that TSC1 deficiency enhances $\mathrm{T}$ cell responses to intestinal antigens. To explore this possibility, the authors used a murine model, in which chronic colitis was induced by exposure to $2 \%$ dextran sodium sulfate (DSS) in the drinking water for 7 days, followed by administration of regular water. In this model, TSC1-deficient mice exhibited more severe weight loss and a slower recovery after DSS removal compared with WT mice. Keeping with the well-recognized pathogenic roles of Th1 and Th17 cells in intestinal inflammation, TSC1-deficient T cells produced excessive amounts of IFN- $\gamma$ and IL-17A. The effect of TSC1 deficiency in promoting Th1 and Th17 differentiation was recapitulated under Th1- or Th17-polarizing conditions in vitro. Furthermore, TSC1-deficient T cells showed enhanced IFN- $\gamma$ and IL-17A production upon antigen immunization in vivo. These findings collectively demonstrate that TSC1 deficiency aberrantly promotes the differentiation of $\mathrm{T}$ cells into Th1 and Th17 subsets. Interestingly, TSC1-deficient $\mathrm{T}$ cells produced normal levels of
IFN- $\gamma$ and IL-17A upon direct ex vivo stimulation, suggesting a specific role for TSC1 in restraining Th1 and Th17 differentiation in inflammatory environments, such as in the intestine or upon immune stimulation (Figure 2).

\section{Maintenance of Treg function and identity}

Intestinal homeostasis arises from a highly dynamic balance between effector $\mathrm{T}$ cell responses and regulatory mechanisms, in which Foxp3-expressing Tregs play a central role (12). Park et al. asked whether exacerbated inflammation in the absence of TSC1 was also due to defects in the Treg compartment, specifically Foxp3 expression and in vivo suppressive activity (11). They observed that Tregs were reduced in the spleens and colonic lamina propria (cLP) of TSC1-deficient mice after DSS exposure. TSC1 deficiency did not impact TGF- $\beta$-mediated generation of induced Tregs in vitro or the homeostasis of thymus-derived Tregs in peripheral lymphoid organs (8). Although previous studies have established a negative role of mTOR signaling in Treg differentiation $(2,5)$, TSC1 deficiency appears to impact the Treg population only under inflammatory conditions. The authors also assessed the suppressive function of TSC1-deficient Tregs in a $\mathrm{T}$ cell-mediated colitis model by transferring conventional $\mathrm{T}$ cells along with WT or mutant Tregs into Rag $1^{-/-}$mice. While the transfer of WT Tregs prevented conventional $\mathrm{T}$ cell-mediated intestinal inflammation, the transfer of TSC1-deficient Tregs did not. To specifically examine the intrinsic role of TSC1 in Tregs, the authors developed a mouse line with
TSC1 specifically ablated in Foxp $3^{+}$Treg cells $\left(\right.$ Foxp $\left.3^{\mathrm{YFPCre}} T s c 1^{\mathrm{f} / \mathrm{f}}\right)$. Tregs from these mice showed impaired suppression of colitis development, similar to that observed in mice with $\mathrm{T}$ cell-specific TSC1 deficiency. These findings indicate that TSC1 is required for maintaining Foxp3 expression and mediating Treg suppression in an inflammatory environment.

What is the underlying mechanism? Under lymphopenic and/or inflammatory conditions, especially in the intestine, Tregs may become unstable, as indicated by loss of Foxp3 expression, and/or aberrantly acquire effector functions, thereby contributing to uncontrolled inflammation (13). Park et al. therefore examined the effect of TSC1 on Treg stability (11). They generated a reporter strain by crossing Foxp $3^{\mathrm{YFPCre}} T s c 1^{\mathrm{f} / \mathrm{f}}$ mice with a mouse strain that expresses RFP as a reporter of Cre activity. In this fate-mapping system, unstable Tregs known as "ex-Tregs" are detected as a unique $\mathrm{RFP}^{+} \mathrm{YFPCre}^{-}$population (i.e., these cells have expressed Foxp3 but are no longer actively expressing Foxp3). Loss of TSC1 expanded the ex-Treg population. More remarkably, this population exhibited a noticeable reduction of Foxp3 expression and acquired the ability to produce IL-17A. These data indicate that TSC1-dependent enforcement of the suppressive activity of Tregs is contingent on the capacity of TSC 1 to maintain

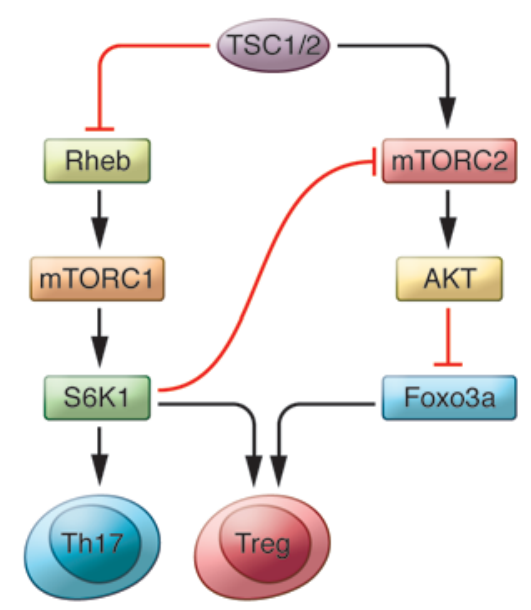

\section{Figure 2}

Roles of TSC $1 / 2$ in Th17 and Treg responses. TSC1 functions within the TSC1/TSC2 complex to repress Rheb-dependent mTORC1 and downstream S6K1 activation. TSC1 also promotes mTORC2/AKT activity to inactivate Foxo3a. TSC1-mediated downstream pathways S6K1 and Foxo3a in turn regulate Th17 and Treg responses. 
Treg stability by preventing diversion into a Th17 effector lineage.

\section{Signaling mechanisms in $\mathrm{T}$ cell fate decisions}

Park et al. explored downstream signaling events in TSC1-deficient T cells to determine TSC1-mediated molecular mechanisms in T cell fate decisions (11). Consistent with previous reports (8-10), TSC1 deficiency promoted mTORC1 activity, as indicated by increased S6K1 phosphorylation and the expression of GRB10, a recently identified mTORC1 target that mediates feedback inhibition of insulin signaling $(14,15)$. The authors then targeted S6K1 and GRB10 to dissect their contributions in Th17 differentiation. Reduction of Grb10 with shRNA had little effect; however, shRNA-dependent knockdown of Rps6kb1 markedly suppressed Th17 differentiation for both WT and TSC1-deficient naive T cells. Rps6kb1 knockdown also prevented increased IL-17A production in TSC1-deficient Tregs. Together, these results demonstrate that S6K1 mediates the effects of TSC1 deficiency and mTORC1 hyperactivation on the promotion of IL-17A production in both conventional $\mathrm{CD}^{+} \mathrm{T}$ cells and Tregs.

Aside from increased mTORC1 activity, TSC1 deficiency attenuated the activation of mTORC2- and AKT-mediated phosphorylation of Foxo1/Foxo3a (8). Attenuated Foxo1/Foxo3a phosphorylation leads to increased stability and function of Foxo1/Foxo3a. However, the importance of this effect remains undefined, since the elimination of Rictor/mTORC2 activity has little effect on naive $T$ cell homeostasis compared with deletion of Tsc1. Foxo family proteins are essential in Treg development and function (16-18); therefore, Park et al. generated mice lacking both TSC1 and Foxo 3 in the Treg compartment (11). Most of the Treg phenotypes in these double-knockout mice were similar to those observed after Tsc1 deletion; however, Foxo3 deficiency further impaired the stability of TSC1-deficient Tregs. Furthermore, these Tregs exhibited accelerated conversion into Th17-like effector $\mathrm{T}$ cells. Thus, the diminished mTORC2 activity in TSC1-deficient Tregs modestly ameliorated the disrupted stability of these cells. Nonetheless, increased IL-17A production in Tregs from both TSC1-deficient and Tsc1/Foxo3a double-knockout mice was blocked by rapamycin treat- ment, highlighting a predominant effect of enhanced mTORC1 activation on the impairment of Treg function and stability (Figure 2).

\section{Perspective}

The intestinal immune system continuously faces innocuous antigens derived from commensal microbiota and diet. Treg cells mediate immune tolerance toward these antigens, preventing effector $\mathrm{T}$ cell activation and undesired intestinal inflammation. TSC1 deficiency in $\mathrm{T}$ cells leads to enhanced Th 1 and Th17 responses, but impaired Treg function and stability under inflammatory conditions such as intestinal inflammation. In contrast, TSC1 function is not required to mediate the balance between effector and regulatory $\mathrm{T}$ cell responses under homeostatic conditions. These results suggest the presence of specific signals during inflammation, especially in the intestine, that sustain TSC1 function in T cells, thereby preventing aberrant $\mathrm{mTORC} 1$ activation and dysregulated effector and regulatory $\mathrm{T}$ cell responses. Antiinflammatory substances such as TGF- $\beta$ and retinoic acid (RA) are important in orchestrating the induction of tolerance in the intestine (12). More recently, short-chain fatty acids, a class of microbial metabolites, have been shown to establish colonic homeostasis via the regulation of Treg generation and function (19). It will be interesting to test whether these tolerogenic substances signal through TSC1 to mediate intestinal homeostasis.

Although mTOR signaling is well recognized as an orchestrator of cell metabolism (20), the metabolic function of $\mathrm{mTOR}$ in $\mathrm{T}$ cells is just beginning to be dissected. Upon antigen stimulation, $T$ cells transit from catabolism to anabolism, with a marked increase in the bioenergetic and biosynthetic demands over the quiescent state. This metabolic reprogramming is considered a crucial checkpoint for $\mathrm{T}$ cell activation and fate decision. mTOR activation is closely associated with the dynamic regulation of $\mathrm{T}$ cell metabolism, but how mTOR signaling intersects with metabolic programs in $\mathrm{T}$ cell responses is poorly understood $(21,22)$. Recently, mTORC1-dependent coupling of immune signals and cell metabolism, especially the lipogenic pathway, was found to be essential for programming Treg suppressive function (23). Interestingly, Park et al. showed that enhanced mTORC1 activity in TSC1-deficient Tregs also impairs their suppressive activity, suggesting that proper control of mTORC1 activity is essential for Tregs to function (11). Whether and how the metabolic programs mediate TSC1 function in Tregs remain to be determined.

The development of potent mTOR inhibitors has been considered an effective therapeutic strategy to treat cancer and other diseases. For example, Torin 1 and PP242 are ATP-competitive inhibitors of $\mathrm{mTOR}$ that strongly suppress mTOR activity (24). However, given the importance of mTORC1 signaling in the function of $\mathrm{T}$ cells and other cells, completely blocking mTOR activity will impair normal immune responses to foreign pathogens. Instead, fine-tuning mTOR activity by targeting context-specific regulators of $\mathrm{mTOR}$ signaling, such as TSC1, offers an attractive strategy to treat autoimmune and inflammatory diseases and restore immune tolerance in the long run.

\section{Acknowledgments}

This work was supported in part by grants from the NIH (AI101407, AI094089, and NS064599), the American Cancer Society (RSG-13-248-01-LIB, to H. Chi), and by a postdoctoral fellowship from the Arthritis Foundation (to K. Yang).

Address correspondence to: Hongbo Chi, Department of Immunology, St. Jude Children's Research Hospital, 262 Danny Thomas Place, Memphis, Tennessee 38105, USA. Phone: 901.595.6282; Fax: 901.595.5766; E-mail: hongbo.chi@ stjude.org.

1. Laplante M, Sabatini DM. mTOR signaling in growth control and disease. Cell. 2012;149(2):274-293.

2. Chi H. Regulation and function of mTOR signalling in T cell fate decisions. Nat Rev Immunol. 2011;12(5):325-338.

3. Lee $\mathrm{K}$, et al. Vital roles of mTOR complex 2 in Notch-driven thymocyte differentiation and leukemia. J Exp Med. 2012;209(4):713-728.

4. Tang F, Wu Q, Ikenoue T, Guan KL, Liu Y, Zheng P. A critical role for Rictor in T lymphopoiesis. JImmunol. 2012;189(4):1850-1857.

5. Delgoffe GM, et al. The mTOR kinase differentially regulates effector and regulatory $\mathrm{T}$ cell lineage commitment. Immunity. 2009;30(6):832-844.

6. Delgoffe GM, et al. The kinase mTOR regulates the differentiation of helper $T$ cells through the selective activation of signaling by mTORC 1 and mTORC2. Nat Immunol. 2011;12(4):295-303.

7. Lee $\mathrm{K}$, et al. Mammalian target of rapamycin protein complex 2 regulates differentiation of Th 1 and Th2 cell subsets via distinct signaling pathways. Immunity. 2010;32(6):743-753

8. Yang K, Neale G, Green DR, He W, Chi H. The tumor suppressor Tsc1 enforces quiescence of naive $T$ cells to promote immune homeostasis and 
function. Nat Immunol. 2011;12(9):888-897.

9. Wu Q, et al. The tuberous sclerosis complex-mammalian target of rapamycin pathway maintains the quiescence and survival of naive T cells. J Immunol. 2011;187(3):1106-1112.

10. O'Brien TF, et al. Regulation of T-cell survival and mitochondrial homeostasis by TSC1. Eur J Immunol. 2011;41(11):3361-3370.

11. Park Y, et al. TSC1 regulates the balance between effector and regulatory T cells. J Clin Invest. 2013; 123(12):5165-5178.

12. Shale M, Schiering C, Powrie F. CD4(+) T-cell subsets in intestinal inflammation. Immunol Rev. 2013;252(1):164-182.

13. Zhou X, et al. Instability of the transcription factor Foxp3 leads to the generation of pathogenic memory $\mathrm{T}$ cells in vivo. Nat Immunol. 2009;10(9):1000-1007.

14. Yu Y, et al. Phosphoproteomic analysis iden- tifies Grb10 as an mTORC1 substrate that negatively regulates insulin signaling. Science. 2011;332(6035):1322-1326.

15. Hsu PP, et al. The mTOR-regulated phosphoproteome reveals a mechanism of mTORC1-mediated inhibition of growth factor signaling. Science. 2011;332(6035):1317-1322.

16. Ouyang W, Beckett O, Ma Q, Paik JH, DePinho RA, Li MO. Foxo proteins cooperatively control the differentiation of Foxp3+ regulatory T cells. Nat Immunol. 2010;11(7):618-627.

17. Ouyang W, et al. Novel Foxo1-dependent transcriptional programs control $\mathrm{T}$ (reg) cell function. Nature. 2012;491(7425):554-559.

18. Kerdiles YM, et al. Foxo transcription factors control regulatory $\mathrm{T}$ cell development and function. Immunity. 2010;33(6):890-904.

19. Smith PM, et al. The microbial metabolites, short- chain fatty acids, regulate colonic Treg cell homeostasis. Science. 2013;341(6145):569-573.

20. Düvel K, et al. Activation of a metabolic gene regulatory network downstream of mTOR complex 1. Mol Cell. 2010;39(2):171-183.

21. Yang K, Chi H. mTOR and metabolic pathways in $\mathrm{T}$ cell quiescence and functional activation. Semin Immunol. 2012;24(6):421-428.

22. Zeng H, Chi H. mTOR and lymphocyte metabolism. Curr Opin Immunol. 2013;25(3):347-355.

23. Zeng H, Yang K, Cloer C, Neale G, Vogel P, Chi H. mTORC1 couples immune signals and metabolic programming to establish $\mathrm{T}(\mathrm{reg})$-cell function. Nature. 2013;499(7459):485-490.

24. Benjamin D, Colombi M, Moroni C, Hall MN. Rapamycin passes the torch: a new generation of mTOR inhibitors. Nat Rev Drug Discov. 2011;10(11):868-880

\title{
Thrombocytopathy and type 2B von Willebrand disease
}

\author{
Jerry Ware
}

Department of Physiology and Biophysics, University of Arkansas for Medical Sciences, Little Rock, Arkansas, USA.

\begin{abstract}
The knowledge gained from "experiments of nature" has always been paramount in identifying key players in pathophysiologic pathways. This is well characterized by naturally occurring bleeding and thrombotic disorders. In most cases, it is the absence of a particular protein that leads to recognition of its importance for normal physiology. On the other hand, gain-of-function mutations highlight not only the presence of the protein, but also how it regulates a particular physiologic response. In this issue of the JCI, Casari and colleagues define a previously unrecognized consequence of variant type $2 \mathrm{~B}$ von Willebrand factor (vWF) binding to blood platelets. More than 30 years after an initial description of type $2 \mathrm{~B}$ variant $\mathrm{vWF}$, the consequence of this spontaneous variant $\mathrm{vWF}$ binding to platelets is viewed as a dysregulation of platelet signaling pathways contributing to the type $2 \mathrm{~B}$ bleeding phenotype.
\end{abstract}

\section{Type 2B von Willebrand disease}

Among bleeding disorders, von Willebrand disease (vWD) is the most common. This inherited disorder is caused by production of a dysfunctional von Willebrand factor (vWF) (1). The current paradigm for the hemostasis regulation is that vWF circulates as a soluble multimeric protein with an indeterminate affinity for platelets or the intact vessel wall (2). Following vascular damage, surface-bound vWF is exposed in the subendothelium and captured from flowing blood to become a key bridging molecule, which supports platelet adhesion to an altered vascular surface. Thus, a simple explanation for the regulation of

Conflict of interest: The author has declared that no conflict of interest exists.

Citation for this article: J Clin Invest. 2013; 123(12):5004-5006. doi:10.1172/JCI73169.
vWF function is that soluble vWF has an unmeasurable affinity for platelets, while surface-bound vWF interacts efficiently with platelets. The importance of vWF and its platelet receptor, glycoprotein Ib-IX (GP-Ib-IX), is highlighted by well-characterized mutations that lead to the absence of vWF or GP-Ib-IX, resulting in bleeding phenotypes $(3,4)$. vWD constitutes a complex spectrum of clinical phenotypes that can be further subclassified based on levels of circulating protein, degree of protein multimerization, and functional interactions with platelets and coagulation factor VIII $(1,3)$.

One of the more intriguing vWD subtypes is the gain-of-function mutation designated vWD-type 2B (5). In this case, single amino acid mutations within the GP-Ib-IX-binding domain of vWF lead to an altered conformation that supports the soluble interaction of vWF with platelets in the circulation
(Figure $1 \mathrm{~A}$ and refs. 6, 7). Because the consequence of type $2 \mathrm{~B} \mathrm{vWF}$ is a spontaneous interaction with circulating platelets, the phenotypic outcome might be expected to be a prothrombotic. Instead, vWD-type $2 \mathrm{~B}$ leads to a counterintuitive phenotype - increased bleeding. The bleeding phenotype is thought to be promoted by the largest vWF multimers, which have the most hemostatic potential, being sequestered by platelets and unable to participate in hemostasis. The presumed consequences of soluble vWF binding to platelets are intravascular platelet clumping, platelet removal from the circulation, and varying degrees of thrombocytopenia. Thus, a combination of the absence of VWF with the most hemostatic potential and thrombocytopenia has historically been the explanation of the type $2 \mathrm{~B}$ bleeding phenotype.

While thrombocytopenia and the absence of high-molecular-weight vWF are reasonable explanations for type $2 \mathrm{~B}$ bleeding, the study by Casari et al. (8) in this issue of the JCI adds an additional explanation. Their current work suggests a third mechanism for type 2B bleeding; thrombocytopathy as a result of dysregulated platelet signaling is a consequence of variant $\mathrm{VWF}$ binding to platelets (Figure 1B). This dysregulated signaling impairs platelet aggregation, platelet secretion, and platelet spreading. For normal hemostasis, each of these platelet events is critical for the temporal sequence of events that support hemostasis. Specifically, Casari et al. provide evidence of 